\title{
Quality Controlled Region-Based Partial Fingerprint Recognition
}

\author{
Omid Zanganeh $^{1}$, Komal Komal ${ }^{2}$, Nandita Bhattacharjee ${ }^{2}$, \\ David Albrecht ${ }^{2}$ and Bala Srinivasan ${ }^{2}$ \\ ${ }^{1}$ Independent consultant, Melbourne, Australia \\ ${ }^{2}$ Faculty of Information Technology, Monash University, Melbourne, Australia \\ E-mail: o.zanganeh@gmail.com (Omid Zanganeh); \\ komal.komal@monash.edu (Komal Komal)
}

Received 24 January 2018; Accepted 4 February 2018;
Publication 24 April 2018

\begin{abstract}
The conventional method of fingerprint alignment using reference points does not work well for partial fingerprints due to the limited or non-availability of reference points. Moreover, matching of partial fingerprints using existing techniques is challenging as partial fingerprints lack enough distinguishing information. Even if fingerprints consists of sufficient information, the varying quality of different parts of fingerprint affects recognition process. In this paper, a new paradigm in the form of region-based approach that uses all available fingerprint ridge structure for aligning the fingerprints is proposed. Additionally, a new metric to compute individual local region similarity based on region's quality, size and consistency of its neighbouring regions is proposed and used in deriving the global similarity for matching process. Although the proposed approach is computationally intensive, yet, the error rate is close to zero as the experimental results shows. The method is most suitable in applications where perfect identification is required such as forensic investigations.
\end{abstract}

Keywords: Partial Fingerprints, Alignment, Region-based, Quality, Similarity Measure, Recognition.

Journal of Mobile Multimedia, Vol. 14_2, 123-156.

doi: 10.13052/jmm1550-4646.1421

(c) 2018 River Publishers 


\section{Introduction}

Matching of partial fingerprints is very essential not only in forensic applications but also in day to day life applications. The fingerprints obtained from the crime scenes are generally low in quality and partial. Moreover, miniaturization of the fingerprint scanner also results in partial fingerprint. The issue of matching the partial fingerprint is similar to full fingerprint but it also has its unique characteristics. The partial fingerprints not only lack enough fingerprint features but also are distorted and unclear. These characteristics of partial fingerprints poses many challenges in implementing the existing fingerprint recognition system. Two major research challenges that need to be addressed are (i) how to align the full and partial fingerprint to simplify the matching process and overcome the rotational difference between the two fingerprints and (ii) how to assign the matching/similarity score to a pair of fingerprints to compensate for high intra-class variation and inter-class similarity.

Before one can measure whether given fingerprints are similar or not, one need to align the given fingerprints. In case of partial fingerprints, only a part of the finger is available and therefore, it becomes challenging to align them before computing their similarity. The previously well developed fingerprint alignment methods, including minutia-based and non-minutia based, may not be suitable for partial fingerprints [1]. One issue of applying these methods to partial fingerprints is that they require features that might not be available in partial fingerprints. Accordingly, these methods will either lead to incorrect alignment or not be applicable [1]. For instance, Khalili et al. have investigated using fingerprint reference points to rotationally align the fingerprints [2]. However, it is likely that reference points are not available in partial fingerprints. Hence, it is difficult to align the partial fingerprints by using the existing methods.

Since the shape and size of the partial fingerprint is not fixed, the fingerprints must be aligned adaptively to the partial fingerprint shape and size. Therefore, it is mandetory to align the partial fingerprints based on the information available in the fingerprints [3]. For that, the pixel based information of the fingerprints can be used for aligning fingerprints. However, in a pixel-to-pixel comparison of two images, even a slight rotational difference between two images might result in assigning a wrong similarity score and consequently a wrong matching decision. In other words, an accurate alignment result in minimizing false decisions in the system. 
Not only the shape and information present in partial fingerprints is an issue but the varying quality in different parts of the fingerprints is also a challenging problem to deal with. The varying quality of different parts of the fingerprint can affect the similarity score and can result in lower or higher similarity score between intra and inter class fingerprints which is not desired. Therefore, the quality of the partial fingerprints need to be compensated for computing the similarity score between them.

In this paper, we propose a region based fingerprint alignment method and a quality controlled similarity measure to compute similarity between fingerprints. The proposed approach focuses on alignment based on correlation of fingerprint's regions. The rotational difference of two fingerprints is obtained even more accurately by using consistency of surrounding regions. The performance evaluation of the proposed method shows that alignment by using only the fingerprint ridges performs slightly better as compared to alignment by using singular points. However, when accuracy is not the main focus of the system, using the singular points (if available) is reasonable due to the faster process of aligning the fingerprints. In addition, before computing the similarity between the fingerprints the quality of the regions is considered to minimize the false matches.

Section 2 discusses the method of aligning fingerprints based on singularity points and fingerprints ridge information. The importance of using ranking and quality of regions for aligning fingerprints is discussed in Section 3. Section 3.1 and 3.2 illustrates the details of ranking and quality of regions for accurate alignment. After aligning fingerprint, the corresponding regions are located by identifying a common off-set point (Section 4). Section 5 discusses the computation of similarity of fingerprints by proposing a regionbased similarity measure. The results of the proposed method are compared with existing methods in Section 6. Section 7 represents the experimental results on two partial fingerprint datasets that shows the effectiveness of the proposed method on partial fingerprints. Experimental results are followed by conclusion in Section 8.

\section{Alignment of Fingerprints}

Alignment of fingerprints is a fundamental step in the fingerprint recognition. Many techniques have been proposed in the literature for aligning the fingerprints. One of the most common approaches is alignment using singularity points. However, fingerprints may not always consist of singularity that makes the alignment process difficult. Therefore, we propose an alignment 


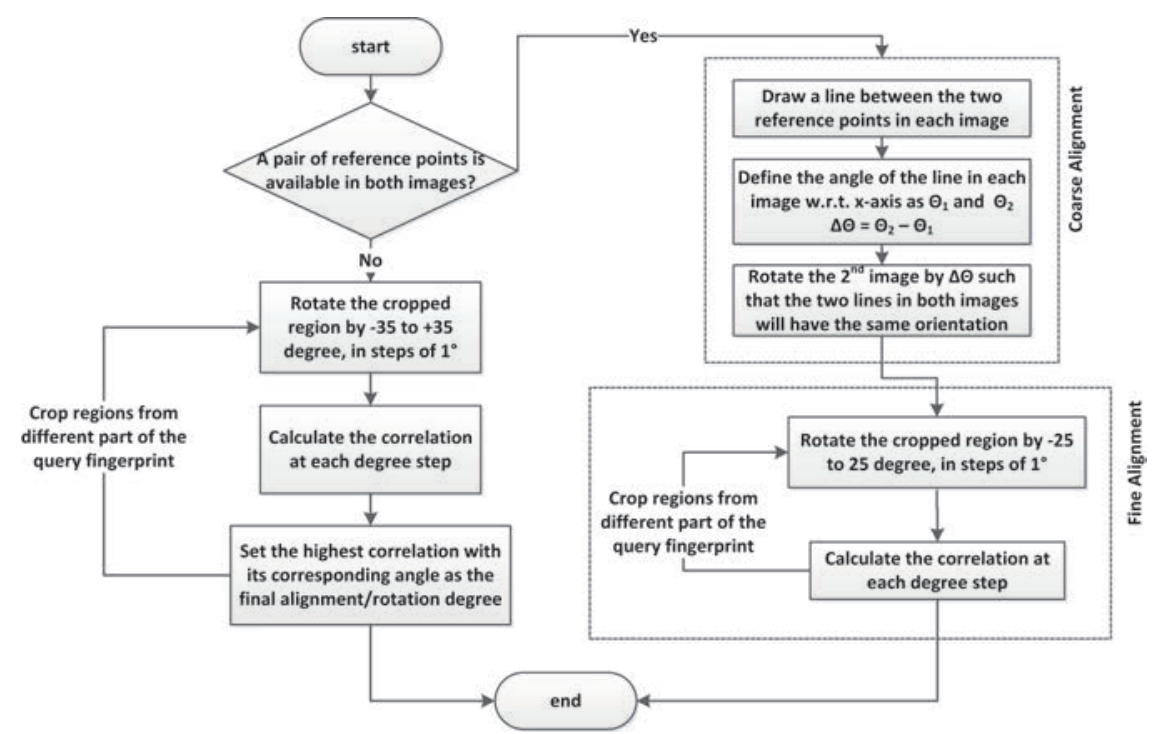

Figure 1 Block diagram of the proposed fingerprint alignment techniques.

method based on ridge information of fingerprint. The proposed method is not only independent of singularities but also result in more accurate alignment. However, if fingerprints consists of singular points, they could be used for fast alignment along with ridges. Singular points can be used to coarsely align fingerprints and ridge structure can be used for fine alignment. The block diagram of ridge based fingerprint alignment with or without using singularity points is presented in Figure 1.

\subsection{Alignment based on Singular Points}

The fingerprints are generally 2-dimensional images which can be aligned if two identical points are located in both images. Singularity points (if available) could be used to align the fingerprints efficiently based on this idea [4]. We have used the method proposed by Wang et al. [5] for detecting singularity points. Assuming that two same reference points exist in both fingerprints, in order to rotationally align fingerprints, query image is rotated till the gradients of the line joining the two reference points in query and registered fingerprints are identical.

Figure 2 illustrates an example of aligning the fingerprints based on the above process. Figures $2 \mathrm{a}$ and $2 \mathrm{~b}$ show registered and query fingerprints respectively, which are different impressions of same finger. To align the 


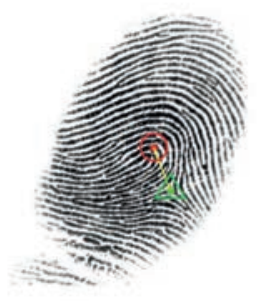

(a)

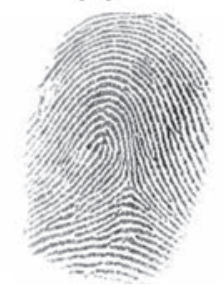

(c)

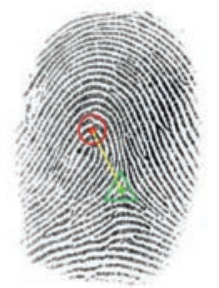

(b)

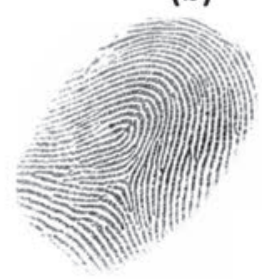

(d)

Figure 2 Aligning the query fingerprint (b) with respect to registered fingerprint (a), in (c) only singular points are used to align the fingerprints, in (d) alignment is done by using both singular points and ridge features.

query fingerprint with registered one, the query fingerprint should be rotated clockwise. The red and green circles depict the location of up-core and delta respectively on both fingerprints. According to the position of the core and delta points on registered and query image, the angle of line joining core and delta with respect to $\mathrm{x}$-axis are $+111^{\circ}$ and $+116^{\circ}$, respectively. This results in the angle difference between two images being $5^{\circ}$. Therefore, the query image should be rotated by $5^{\circ}$ (clockwise) to be aligned with the registered image (Figure 2c).

Using singularity points for alignment has mainly two issues: (i) the intra-class variation makes location of the singularity points vary from one fingerprint to another which may result in inaccurate alignment; and (ii) detecting the location of singularity points accurately is very challenging [4]. Detection of singular points depends on the method applied to identify the singular point location [5]. However, this alignment method can be used for a coarse alignment when the efficiency is more important than accuracy. In order to align the fingerprints accurately and efficiently, the singularity based alignment can be combined with ridge based alignment (as discussed in next section). 


\subsection{Alignment based on Fingerprint Ridge Structure}

Aligning fingerprints based on ridge structure is not dependent on fingerprint's shape, size and singularity points. As long as a fingerprint is provided, it contains the fingerprint ridge (even if it is a small area) and that can be used to align the fingerprints. The alignment process starts with cropping a region from query fingerprint. The size of cropped region can vary depending on size of valid fingerprint area. Considering the size of fingerprints in dataset FVC_2002, we set the size of the cropped regions to $128 \times 128,128 \times 64$, and $64 \times 64$ pixels. Figure 3 shows query fingerprint that is decomposed into regions of size $64 \times 64$ which provides simple extraction of region with bigger sizes (by concatenating the adjacent regions). The regions that are fully occupied with fingerprint information (not part of the background) are selected for alignment. In addition, the region can be cropped from different parts of query fingerprint and can be of different sizes. These two properties make this strategy suitable for partial fingerprints alignment.

For each cropped region, the correlation of cropped region and registered fingerprint is computed for different rotation angles. The rotation angle which gives the highest correlation value, is used to rotationally align two fingerprints. This process can be used even when partial fingerprints do not consist of singularity point. Aligning fingerprints by this method reasonably takes care of finger skin elasticity and effect of distorted regions, as different regions (with different sizes) can be cropped from the query fingerprint.

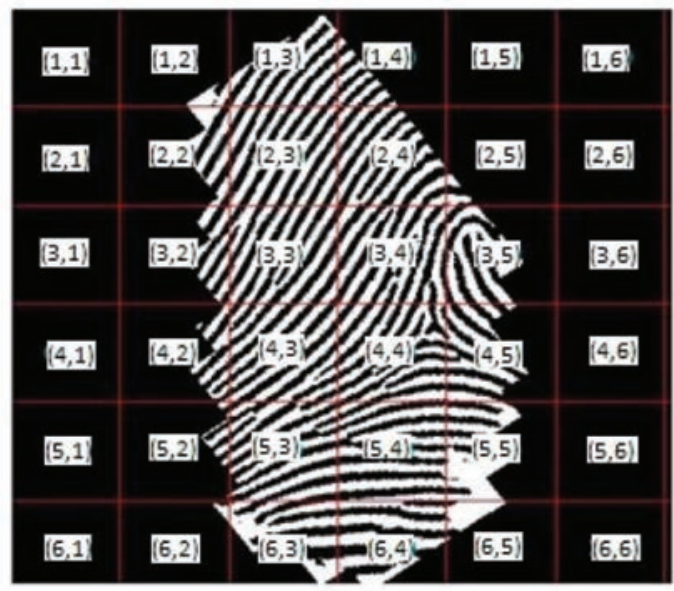

Figure 3 A query fingerprint decomposed into sub-regions $[(x, y)$ is used as sub-region index]. 


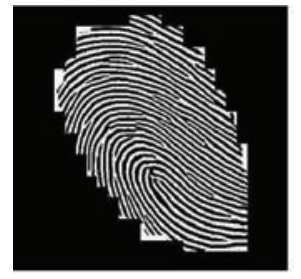

(a)

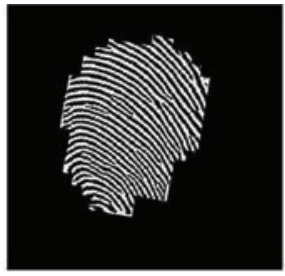

(b)

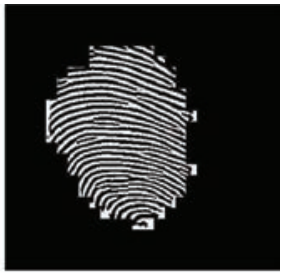

(c)

Figure 4 Impressions of the same finger being aligned based on computing the correlation of their ridge structure. a) registered fingerprint b) query fingerprint c) aligned query fingerprint.

The result of aligning by this strategy is shown in Figure 4. This strategy is accurate but not efficient in terms of computational cost. This is because, each cropped query region is rotated by -35 to +35 (it is the highest rotation difference as mentioned in the FVC_2002 dataset description) degrees till the rotation difference is found which makes the whole process exhaustive.

In order to increase efficiency, singular points position (if available) on fingerprint can be used along with ridges as the second strategy. For that, fingerprints are first coarsely aligned by using the singularity point (as discussed in last section) and then finely aligned by rotating each query region for each angle between -25 to +25 degrees (as fingerprints are already coarsely aligned so do not need to rotate query fingerprint for all angles between -35 to +35 ) and computing the correlation with all registered image regions. Figure 1 depicts the alignment processing using both singularity point and ridge structure and the result of aligning the fingerprints by using singularity and ridge together is shown in Figure 2.

\section{Effect of Ranking and Quality of Regions}

As we have discussed in the Section 2, query regions are rotated and correlation is computed with registered image regions. The registered image region that results in maximum correlation is taken as the matching region. However, the issue is that the highest correlation value achieved (through overlapped regions) might not always be the correct value to select (as illustrated in Figure 5). The presence of more than one peak (highest correlation by overlapping two regions) with approximately the same value shows the probability of choosing the wrong peak. Therefore, choosing the region resulting in highest peak may result in inaccurate alignment. Whereas if the second highest correlation value is considered by taking into account the surrounding regions, 


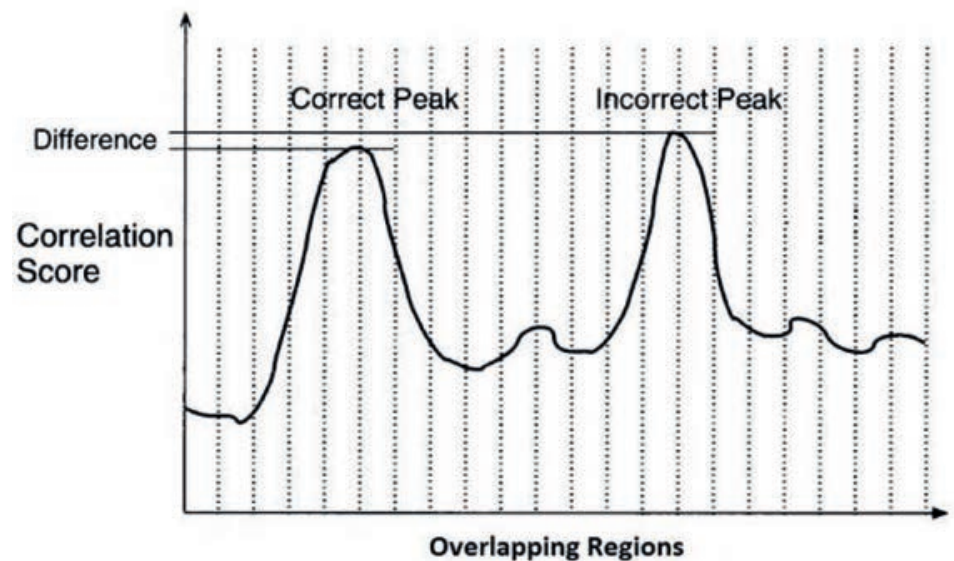

Figure 5 Correlation score of overlapping a region on another image [6].

the result might be more accurate as it corresponds to the global structure of the fingerprint.

Figure 6 illustrates a situation where highest peak does not result in assigning highest possible similarity to intra comparisons. Correct and incorrect peaks should be defined according to global structure of the fingerprint that is measured by considering other available regions in query and registered fingerprint. Thus, in order to improve the alignment by using the ridges, Section 3.1 discuses how to use the global fingerprint ridge structure.

In addition, the quality of captured fingerprints varies because of distortion and other intra-class variations. Not only different impressions of same finger have different quality but also different regions of same fingerprint vary in quality. The low quality/highly distorted regions can be ignored in order to make sure that reliable information is obtained by comparing fingerprint regions. Section 3.2 discusses the method of measuring the quality of regions.

\subsection{Ranking Strategy based on Global Structure}

To make sure the angle selected for aligning the fingerprints is accurate, the location and direction of other regions in neighbourhood are taken into account. According to the information provided by other regions cropped from the query fingerprint, reliability of the highest peak obtained from overlapping the query region on the registered fingerprint can be assessed. Based on assessment, a ranked value is assigned to the cropped region in 

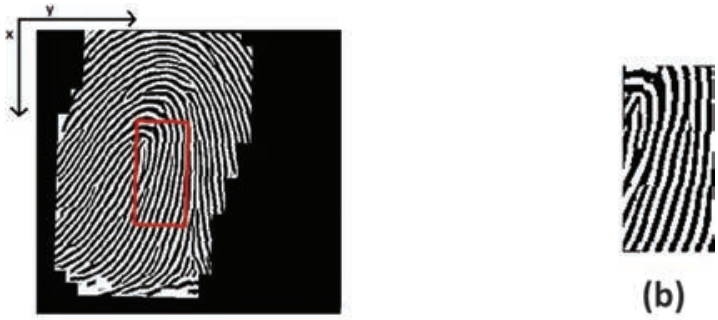

(a)

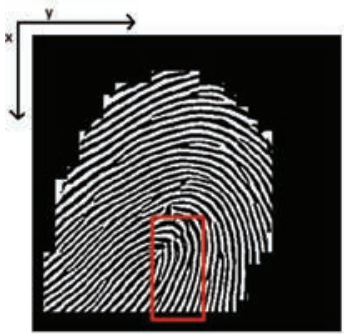

(b)

(c)
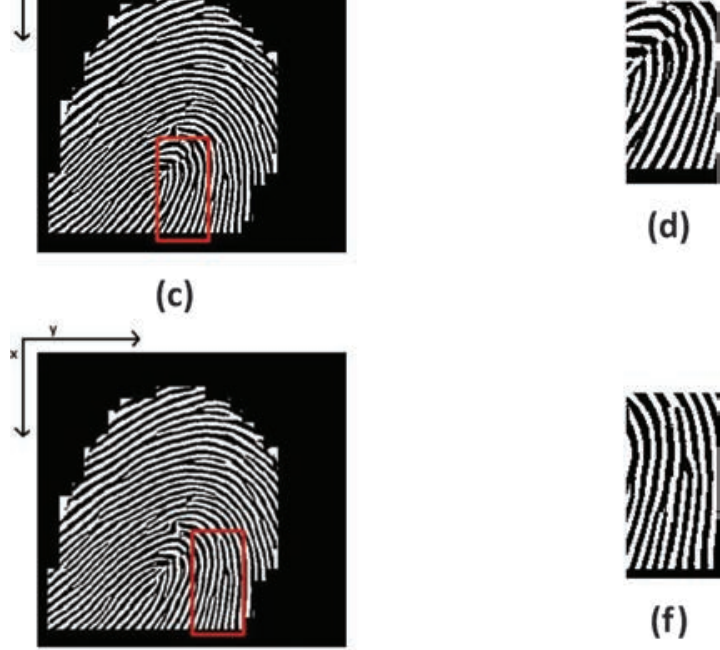

(d)

(e)

Figure 6 a) Query fingerprint. b) cropped region from query fingerprint. c) and e) registered fingerprint. d) the region on registered fingerprint that gives the highest $\mathrm{NCC}$ value f) the region on registered fingerprint that gives the second highest $\mathrm{NCC}$ value.

order to compare it with other regions and align the fingerprints based on the most reliable (highest ranked) region. The ranking is performed based on location and direction of other regions that have been cropped from the query fingerprint and overlapped on the registered image.

Figure 7 shows an example of the available cropped regions from a query fingerprint (indicated as $\mathrm{A}, \mathrm{B}, \mathrm{C}$ ) and their corresponding regions on a registered fingerprint (A', $B$ ' and $C$ ' respectively). If " $A$ " is the region to be ranked, the angle and distance differences of A and B versus $\mathrm{A}^{\prime}$ ' and $\mathrm{B}$ ', 


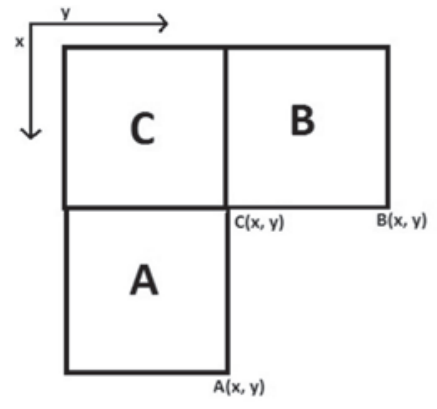

(a)

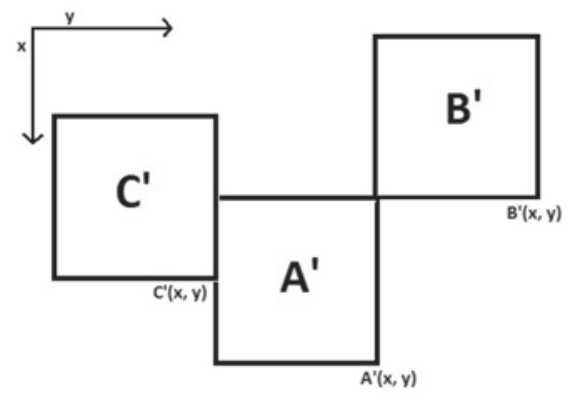

(b)

Figure 7 An example of a) three regions cropped from query fingerprint and b) their corresponding regions on registered fingerprint.

$A$ and $C$ versus $A^{\prime}$ and $C^{\prime}$ is computed. For instance, the angle and distance differences of A and B versus $\mathrm{A}^{\prime}$ and $\mathrm{B}^{\prime}$ is computed as:

$$
\begin{aligned}
& \text { Euclidean Distance }_{(A, B)}=\sqrt{\left(A_{x}-B_{x}\right)^{2}+\left(A_{y}-B_{y}\right)^{2}} \\
& \text { Euclidean Distance }_{\left(A^{\prime}, B^{\prime}\right)}=\sqrt{\left(A_{x}^{\prime}-B_{x}^{\prime}\right)^{2}+\left(A_{y}^{\prime}-B_{y}^{\prime}\right)^{2}} \\
& \text { Difference of Euclidean Distance }\left(\text { AandB }_{\text {vs }} A^{\prime} \text { and }^{\prime}\right) \\
& =\text { Equation } 1-\text { Equation } 2 \\
& \theta_{1}=\tan ^{-1}\left(\frac{A_{x}-B_{x}}{A_{y}-B_{y}}\right), \quad \theta_{2}=\tan ^{-1}\left(\frac{A_{x}^{\prime}-B_{x}^{\prime}}{A_{y}^{\prime}-B_{y}^{\prime}}\right) \\
& \text { Direction }_{\left(\text {sub-region }_{A a n d B}, \text { sub-region }_{\left.A^{\prime} \text { and } B^{\prime}\right)}\right.}=\left|\theta_{2}-\theta_{1}\right|
\end{aligned}
$$

If Euclidean distance and direction difference for each pair of sub-regions is smaller than the Threshold 1 for distance and Threshold 2 for angle respectively, the pair of sub-regions are considered consistent. Threshold 1 is set to 20 pixels and Threshold 2 is set to 25 degrees, as our experiments shows that this setting outputs the best matching results.

The ranking assigned to region $A$ is based on the ratio of the other neighbouring sub-regions that pass the above two conditions. Ranking based on the ratio of the other regions makes the ranking strategy flexible in the case of partial fingerprinting as the number of other available regions may vary. Thus, the ranking assigned to regions ${ }_{A}$ is as follows:

$$
\operatorname{Rank}_{\left(\text {region }_{A}\right)}=S_{A}+S_{A} \times\left(N_{A} / A S_{A}\right)
$$


where $\mathrm{S}_{A}$ represents the similarity (correlation value), $\mathrm{N}_{A}$ represents the number of consistent regions and $\mathrm{AS}_{A}$ represents the number of available regions.

In addition to the number of other available regions, their size also need to be considered in the ranking as one can choose different sized regions. Due to the intra-class variations and image distortion, regions of bigger size have less chance in finding the match in the registered fingerprint and result in a high similarity value. Thus the ranking is compensated by taking care of the region sizes as:

$$
\operatorname{Rank}_{\left(\text {region }_{i}\right)}=S_{i}+S_{i} \times\left(N_{i} / A S_{i}\right) \times S W_{i}
$$

where $S W_{i}$ represents the weight assigned to regions according to their size. The bigger the sub-region size is, the bigger weight is assigned to it. In our calculations, we have used three different sized regions of sizes $64 \times 64$, $64 \times 128$ and $128 \times 128$. After ranking each sub-region according to the Equation (7), the rotational difference of the two fingerprints is detected based on the angle value provided by the region that has the highest ranking. Table 1 shows the results of overlapping a query region on two different regions of a registered fingerprint. As indicated by the "number of available regions" in the table, there are 3 and 6 available regions that could be used to validate the reliability (assign ranking) of region 1 and region 2 of registered fingerprint respectively. For each one of these 3 and 6 other regions, the $(x, y)$ location of where the highest correlation is achieved is recorded. Based on Equations (3) and (5), region 1 and region 2 are having 0 and 4 consistent regions. This results in ranking for region 2 (0.5367) more than that of region 1 (0.4424) whereas the correlation value of region 1 is more than region 2. Hence, the global structure of the fingerprint helps in selecting the correct angle required to align the fingerprints.

Table 1 Result of overlapping cropped region from query fingerprint on two regions from registered fingerprint

\begin{tabular}{lcc}
\hline & Region 1 & Region 2 \\
\hline angle & -9 & 6 \\
\hline correlation value & 0.4424 & 0.4128 \\
\hline Number of regions showing consistency & 0 & 4 \\
\hline Number of available regions & 3 & 6 \\
\hline (x, y) of cropped region from query fingerprint & $(256,192)$ & $(256,192)$ \\
\hline (x, y) of cropped region from registered fingerprint & $(356,216)$ & $(352,259)$ \\
\hline Ranked value & 0.4424 & 0.5367 \\
\hline
\end{tabular}


Table 2 Recognising all the fingerprints only based on alignment information on $F V C \_2002 \_D B 1$

\begin{tabular}{lcc}
\hline Training set size $(\%$ of the dataset): & Accuracy $(\%)$ & Error Rate(100 - Accuracy) $(\%)$ \\
\hline $100+99(\sim 2.5 \%)$ & 89.8 & 10.2 \\
\hline $200+197(\sim 5 \%)$ & 94.6 & 5.4 \\
\hline $300+249(\sim 7 \%)$ & 94.5 & 5.5 \\
\hline
\end{tabular}

\subsubsection{Recognition based on ranking score}

The information obtained by ranking regions can be used to recognise the fingerprints without any further processing (results are shown in Table 2). The alignment and ranking of regions according to the other regions (Section 3.1) provides useful information that can be used to recognise fingerprints with no need to detect common regions and compute local and global similarities. Recognizing fingerprints at this stage will improve system efficiency since many of the comparisons can be recognised to be from the same finger (genuine user) or not (imposter user) without any further processing. The table shows that by increasing the training set size upto 7\%, we can obtain accuracy of $94.5 \%$.

\subsection{Quality Estimation of Regions}

One of powerful measures to estimate the clarity of ridges and valleys in a fingerprint is orientation coherence [4, 7]. Ridge orientation refers to the angle that a ridge crosses through in a small neighbourhood with a horizontal axis. In order to compute the orientation coherence, the image is partitioned into regions of size $b \times b$. For each block $B, g_{s}=\left(g_{s}^{x}, g_{s}^{y}\right)$ denotes the gradient of grey level intensity at site $s \in B$. The covariance matrix of the gradient vectors of the grey level intensities for all the pixels in the region $s$ is given by [7]:

$$
J=\frac{1}{b^{2}} \sum_{s \in B} g_{s} g_{s}^{T} \equiv\left[\begin{array}{ll}
j_{11} & j_{12} \\
j_{21} & j_{22}
\end{array}\right]
$$

The orientation coherence based on the above symmetric positive matrix is:

$$
\begin{aligned}
& \lambda_{1}=\frac{1}{2}\left(\operatorname{trace}(J)+\sqrt{\operatorname{trace}^{2}(J)-4 \times \operatorname{det}(J)}\right) \\
& \lambda_{2}=\frac{1}{2}\left(\operatorname{trace}(J)-\sqrt{\operatorname{trace}^{2}(J)-4 \times \operatorname{det}(J)}\right)
\end{aligned}
$$


where $\operatorname{trace}(J)=j_{11}+j_{22}, \operatorname{det}(J)=j_{11} \times j_{22}-j_{12}^{2}$ and $\lambda_{1} \geq \lambda_{2}$. The normalized Coherence $(C)$ measure is computed as:

$$
C=\frac{\left(\lambda_{1}-\lambda_{2}\right)^{2}}{\left(\lambda_{1}+\lambda_{2}\right)^{2}}=\frac{\left(j_{11}-j_{22}\right)^{2}+4 j_{12}^{2}}{\left(j_{11}+j_{22}\right)^{2}}
$$

In the above equation, $0 \leq C \leq 1$. If the ridges and valleys in the region are distinguished clearly, then $\lambda_{1} \gg \lambda_{2}$ resulting in $C \approx 1$. On the other hand, if the region is of low quality and the ridges and valleys are not clearly separated, $\lambda_{1} \approx \lambda_{2}$ resulting in $C \approx 0$ [7]. Hence, the coherence value is used as a quality measure for regions. In other words, considering that $j_{11}=g_{x}^{2}$, $j_{12}=j_{21}=g_{x} g_{y}$ and $j_{22}=g_{y}^{2}$, Equation (11) will be one or zero if the values of covariance matrix $J$ are:

$$
\begin{array}{lll}
C=1, & \text { if } & j_{12}^{2} \approx j_{11} \times j_{22} \\
C=0, & \text { if } & j_{12}^{2} \ll j_{11} \times j_{22}
\end{array}
$$

The regions near the fingerprint singularities are likely to provide more discriminative information than the peripheral regions. However, orientation coherence fails to measure the quality near the singularities when there is high curvature which results in low coherence [4]. Figure 8 shows the result
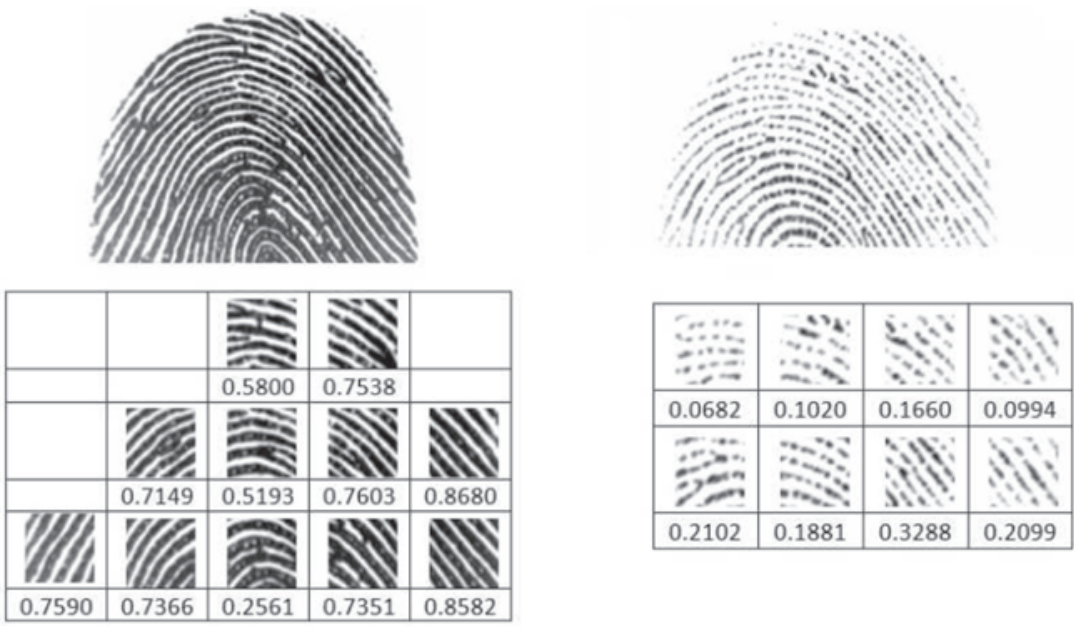

(a)

(b)

Figure 8 a) a partial fingerprint of good quality and b) poor quality. Orientation coherence metric is shown for each region. 
of computing the orientation coherence on different regions of two good and poor quality fingerprints. In Figure 8(a), the region containing high curvature results in low orientation coherence. In Figure 8(b), the orientation coherence is low for almost all the regions due to the low quality of these regions.

\subsection{Alignment of Fingerprints using Ranking and Quality of Regions}

Figure 9 illustrates the process of ridge based alignment of fingerprints using ranking and quality of regions. Considering the intra-class variations and different quality in different regions of the fingerprint, the images are

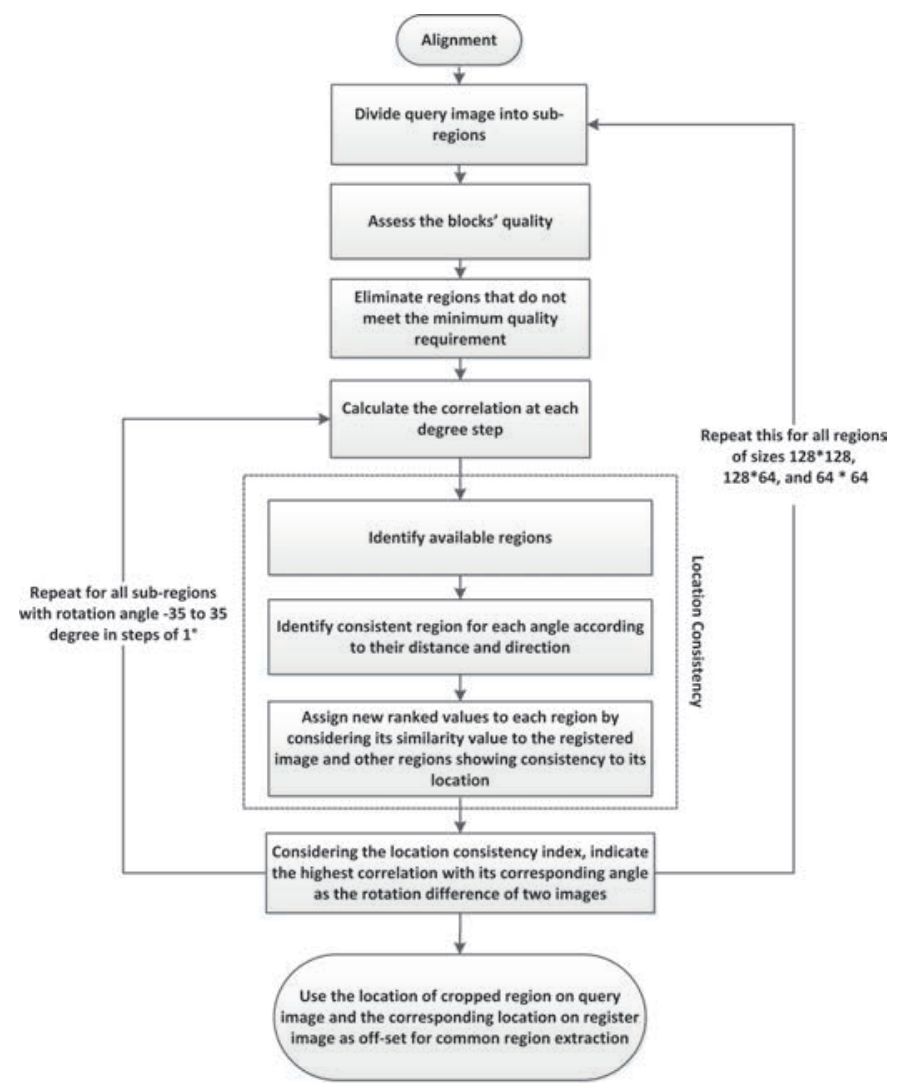

Figure 9 Block diagram of the improved alignment method based on ranking and quality of the regions. 
decomposed into regions. In addition, at this stage, it is not clear which regions in the query fingerprint are available in the registered fingerprint. It emphasizes the need to decompose fingerprint into regions and treat each one of the regions separately to find its match in the registered image. Thus, fingerprints are treated locally by dividing them into regions as indicated in Figure 3. After the query fingerprint is divided into regions, the low quality/highly distorted/not fully contained regions are ignored in order to make sure that reliable information is obtained from fingerprint. Then, each query region is overlapped with the registered fingerprint to obtain the initial value of rotation difference between two fingerprints.

In the previous sections, how the regions are ranked in order to identify a region that shows the highest consistency with other available regions is presented. The ranking strategy is used to identify the matching registered image regions. The registered image region that results in highest rank with query region is used for: (i), rotating the query fingerprint to align with registered fingerprint; and (ii), the coordinates of registered and query fingerprint region are used as the off-set point to extract the common region between the two fingerprints which is discussed in Section 4.

\section{Common Region Extraction for Comparison}

Extracting the common regions between the fingerprints is required to compute the similarity between fingerprints. Fingerprint singularity points can be used to extract the corresponding common regions between the fingerprints. However, singularity points existence cannot be guaranteed in partial fingerprints. Thus, the information provided by fingerprint regions (used for aligning the fingerprints) is used to extract the common regions. The common/corresponding regions of the 2-dimensional fingerprints could be detected by determining an off-set point. Based on the ranking strategy mentioned before, the most reliable region is selected and the location of the overlapping region with the maximum correlation among the other regions is used as the off-set to detect the corresponding regions. Corresponding regions are identified based on the distance of each region on the registered and query fingerprint to the off-set point.

Identifying the corresponding regions in this manner overcomes the translation difference in two images. It should be mentioned that in this case, the common regions between the two fingerprints are detected independent of any particular feature such as singular points making it suitable for partial fingerprints since a small region is enough to complete the process of alignment and common region extraction. 


\section{Similarity Computation}

We need to compute the similarity between the fingerprints in order to aid the matching process. Due to the small overlap of partial query fingerprint with the registered fingerprint, determining the similarity can be very challenging. Therefore, we need a similarity score measurement that can take into account this small overlap resulting from partial fingerprint matching. Measuring the similarity score based on correlation coefficient is promising since it reflects all the available features. In addition, the fingerprints could suffer from distortion that may not be uniformly distributed and different regions may have different image quality levels. Thus, computing the similarity of the fingerprints locally reduces the effect of the distorted regions on the overall similarity score. As a consequence, considering the quality of sub-regions can result in better discrimination between inter and intra cases.

Two intra fingerprints (with low intra-class variation) should have high correlation in every block and vice versa for inter fingerprints. However, one of the main reasons that intra fingerprints result in low similarity is that some parts of the fingerprints are distorted. Therefore, identifying the low quality regions and reducing their effect on the final similarity helps to increase the similarity of intra cases and reducing the probability of falsely rejecting them. On the other hand, by so doing, the final similarity of inter cases will not be affected significantly since the low similarity in inter cases is mainly due to the difference between ridges and valleys structure of the two fingerprints not because of their quality.

Cappelli et al. in [8] claimed that in recent decades local matching has addressed the weaknesses in global matching such as high computational complexity and lack of robustness with respect to non-linear distortion. In next section, how local similarities are computed and how they tolerate the effect of distorted regions on the fingerprint similarity score is discussed. Additionally, the global similarity score is computed for comparison, by consolidating local similarities. The selected local similarities through global consolidation techniques are then averaged by different averaging techniques.

\subsection{Local Similarity Scores}

Conventionally, in correlation-based methods, whole fingerprint is considered as one big single-region. Accordingly, the similarity score is computed based on the correlation of the two single-regions. In order to lower the effect of distorted regions on fingerprints, the similarity of two fingerprints is computed locally. Computing the similarities of each pair of small-regions 
individually and then averaging them to obtain the global similarity is a robust and reliable technique for partial fingerprint matching since the final similarity score is computed by considering the same contribution to each of the local similarities. By doing so, the distorted regions are taken into account as individual local similarity values and it does not affect the similarity of other regions.

Local similarities are computed based on the Normalized Cross Correlation (NCC) of a pair of corresponding regions. The NCC of 2-dimensional images (the two corresponding regions) $f$ and $t$ is calculated as (aka Pearson's r correlation) [9]:

$$
N C C=\frac{1}{n} \sum_{x, y} \frac{(f(x, y)-\bar{f})(t(x, y)-\bar{t})}{\sigma_{f} \sigma_{t}}
$$

where $\bar{f}$ and $\bar{t}$ are mean and $\sigma_{f}$ and $\sigma_{t}$ are standard deviation of images $f$ and $t$ respectively and $n$ is the number of pixels of the images. $x$ and $y$ represent the coordinates of the pixels in the two dimensional $f$ and $t$ fingerprints.

After the local similarities are computed, a particular value (a global score) needs to be obtained from the local similarities to indicate their overall similarity score [8]. To obtain the final similarity score between the two fingerprints (global score/similarity), the local similarities are further processed by averaging methods and global consolidation techniques.

\subsection{Global/Final Similarity Score}

Consolidating the results of local matching into a single value representing the global/final similarity of the fingerprints is challenging since it could highly affect the system performance. Cappelli et al. [8] introduced four global score consolidation techniques. Further to the global consolidation technique proposed by Cappelli et al., one of the widely-used techniques is averaging the local similarities by computing the arithmetic mean.

\subsubsection{Global similarity consolidation methods}

Cappelli et al. [8] introduced four global consolidation techniques based on their local similarities. Although these techniques were used in minutiaebased matching it could also be extended to region-based matching with some modification. Local Similarity Assignment (LSA) and Local Similarity Assignment with Relaxation (LSA-R) techniques search for the best match between minutiae points. This could lead to the maximum local similarities 
which also compensates for the fingerprint alignment. However, in the proposed method the fingerprints are aligned that make these two techniques not applicable.

Local Similarity Sort (LSS) technique sorts all the local similarities and selects the highest $n(n>0)$ similarities. Instead of selecting a constant $n$ number of local similarities, the top $k$ percent also can be selected. This provides more flexibility in partial fingerprint matching since the number of local similarities vary according to the available portion of the fingerprint area. This could also be applied by using all the available information by setting $n$ to the maximum number of available local similarities. The global score is then calculated by averaging the corresponding local similarities (Section 5.3).

Local similarities are sorted by considering the quality of each region in the query fingerprint and its pair in the registered fingerprint. The sorting is done according to the mean and standard deviation $(s t d)$ of the quality of the regions as:

$$
\text { QualityRank }=\frac{\operatorname{mean}(a, b)}{\operatorname{std}(a, b)},
$$

where $\mathrm{a}$ and $\mathrm{b}$ are the quality of the regions computed as discussed in Section 3.2. According to this Equation (13), the numerator and denominator make sure that if both regions $a$ and $b$ are of good quality, they will be assigned to a high quality rank. However, if one is of low quality, the standard deviation will be higher which results in a lower quality rank. By sorting the local similarities according to Equation (13), local similarities that are obtained from the highest quality regions can be selected. This results in local similarities that are more reliable.

In Local Similarity Sort with Relaxation (LSS-R) technique if a local similarity is surrounded by regions producing higher similarity, then its score is strengthened. Here, surrounding regions are also of high quality, or the comparison is an intra case from which a larger region with high similarity can be detected in both query and registered fingerprints. This technique is more flexible to the number of neighbouring regions due to the different number of local similarities as well as different number of neighbour regions on the borders. Like LSS, the selected similarities can then be averaged.

\subsection{Averaging Methods}

The concept of averaging is simple, but it is essential to know which average to use. Choosing the right averaging method enables correct estimation of the central tendency of the population. To analyse the effect of averaging, 
three different averaging methods, namely harmonic, geometric and arithmetic mean are applied to calculate the global similarities from local similarities selected by LSS and LSS-R techniques.

The common merit between all these three means is that they all are calculated based on all the observations [10]. Thus, these three means meet the requirement of giving contribution to all the available information in the fingerprint. However, the main difference between these means is how they are affected by extreme values. Out of these three, geometric mean is the least affected by data skew followed by arithmetic mean and harmonic mean [10]. It is essential to determine whether being affected by extreme values leads to better discrimination of inter and intra fingerprints or not (Section 5.4).

Averaging the correlation values may be biased due to skew in data distribution. The skew in data distribution in proposed method refers to low local similarities when the majority of local similarities are high and vice versa. The skew of correlation values occurs due to the intra-class variation and inter-class similarity of fingerprints. In the proposed region-based method, the intra-class variation and inter-class similarity can be referred to as low local similarities in intra comparisons and high local similarity in inter comparisons.

\subsubsection{Fisher transformation}

To lower the effect of skewed local similarities the Fisher transformation technique [11] can be applied to transform the data into an almost Gaussian Probability Density Function (PDF). Fisher transformation normalizes the distribution of Pearson's $r$ correlation and can be used to obtain an average value that is less affected by distribution skew in fingerprint matching. The Fisher transformation is obtained as:

$$
z=0.5 \times \ln \left(\frac{1+r}{1-r}\right),
$$

where $r$ is the local similarity. The global similarity score is then computed by taking the mean value of $z$ (as transformed values). After averaging, an inverse of Fisher transform is applied to normalize the mean value of $r$ 's for the global similarity score of the two fingerprints to be in range of $(-1,+1)$. The inverse transformation is achieved by:

$$
\bar{r}=\frac{e^{2 \bar{z}}-1}{e^{2 \bar{z}}+1}
$$

Figure 10 shows the correlation coefficient (ranges from -1 to +1 ) and their corresponding value after being transformed by Fisher's technique (ranges 


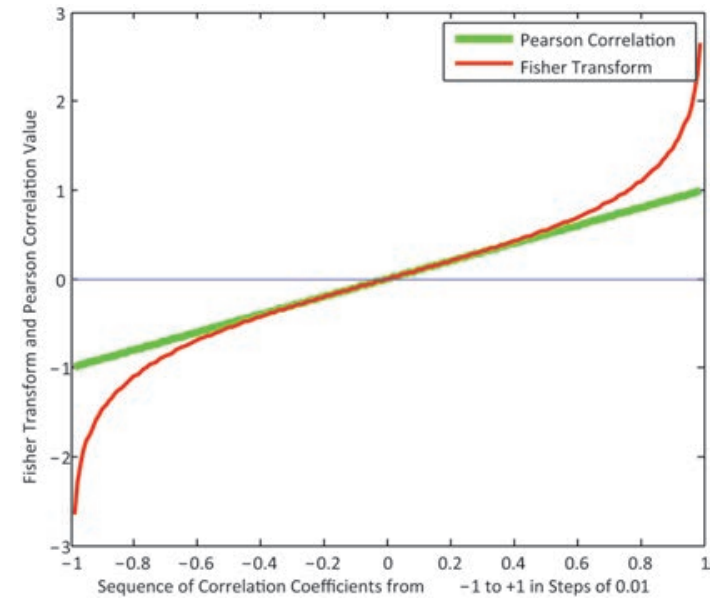

Figure 10 The transformation of the Pearson Correlation (green line) by Fisher Transform Technique (red curve).

from $-\infty$ to $+\infty$ ). As shown, if the correlation coefficient is greater than zero, the value is increased after transformation. Therefore, averaging the transformed data (global score before inverse transformation is applied) is higher than the average of the correlation coefficients. This is desired since it can reduce the effect of distorted regions on the final global score of matching. In other words, if only a few regions are distorted on a fingerprint, low correlation coefficients will be obtained as their local similarities will be normalized.

In case of an intra comparison, most of the correlation values for a pair of corresponding regions (local similarities) indicate high similarity while a few of them indicate low similarity due to low quality. It is likely that this comparison will be falsely rejected if the average of local similarities are used as the global score. However, by applying Fisher's transformation technique, the global score is higher than normal averaging. This is desirable for intra comparison since a higher global score is obtained compared to averaging the correlation values.

\subsection{Experimental Comparison of Different Averging Methods}

To evaluate the effect of the global consolidation techniques and averaging methods, experiments are conducted on the dataset FCV_2002_DB1. Table 3 shows the performance of the proposed partial fingerprint matching using LSS 
Table 3 Comparison of the performance of the partial fingerprint matching by applying different global similarity consolidation and averaging techniques on the dataset FVC_2002_DB1 in terms of the metrics $\operatorname{EER}(\%)$

\begin{tabular}{lllllll}
\hline \multirow{2}{*}{\begin{tabular}{l} 
Global $\begin{array}{l}\text { Consolidation } \\
\text { Technique }\end{array}$ \\
\cline { 2 - 7 }
\end{tabular}} & $\begin{array}{l}\text { A.M. } \\
\text { (Fisher) }\end{array}$ & A.M. & $\begin{array}{l}\text { H.M. } \\
\text { (Fisher) }\end{array}$ & H.M. & $\begin{array}{l}\text { G.M. } \\
\text { (Fisher) }\end{array}$ & G.M. \\
\hline LSS, $\mathrm{n}=100 \%$ & 2.6650 & 2.8150 & 3.0703 & 3.0714 & 2.8429 & 2.8531 \\
\hline LSS, $\mathrm{n}=90 \%$ & 2.7871 & 2.8531 & 3.0702 & 3.0714 & 2.8803 & 2.8834 \\
\hline LSS, $\mathrm{n}=80 \%$ & 2.8632 & 2.9292 & 3.0191 & 3.0232 & 2.9366 & 2.9572 \\
\hline LSS-R & 2.8334 & 2.9196 & 3.1519 & 3.1683 & 2.9689 & 2.9779 \\
\hline
\end{tabular}

and LSS-R global consolidation techniques and using arithmetic, harmonic, and geometric mean (also combined with Fisher transformation) to obtain the final similarity of the two fingerprints. The arithmetic mean is less affected by extreme values than the harmonic mean with the geometric mean least affected.

As indicated in Table 3, both of the global consolidation technique and averaging methods are important and affect the final result of recognition accuracy. The best result is obtained when all the local similarities are taken into account to compute the final similarity value (LSS, $\mathrm{n}=100 \%$ ). Likewise, by using lesser number of local similarities (LSS, $\mathrm{n}=90 \%$ and LSS, $\mathrm{n}=80 \%$ ) the equal error rate (EER) increases. As stated in Section 5.3, applying Fisher transformation on NCC values could lead to reducing the bias in averaging. Regarding the outliers in local similarities, it is worth mentioning that by applying quality measurement techniques they are already ignored and the highly distorted regions do not participate in the final similarity score.

\section{Evaluation of Matching Process}

The block diagram of the overall region based fingerprint matching approach is presented in Figure 11. This diagram explains the use of ranking and quality of regions for aligning the fingerprints and the method of finding the corresponding matching regions between the fingerprints. Also, illustrates the process of computing similarity between fingerprints by applying Fisher transform, Local Similarity Sort and averaging methods. Based on the similarity value of fingerprints, a decision is made for matching.

Experiments for evaluating the proposed approach are conducted on public datasets FVC_2002_DB1 and FVC_2006_DB2 [12]. Table 4 shows the description of the datasets. As discussed in [12], to collect the dataset 


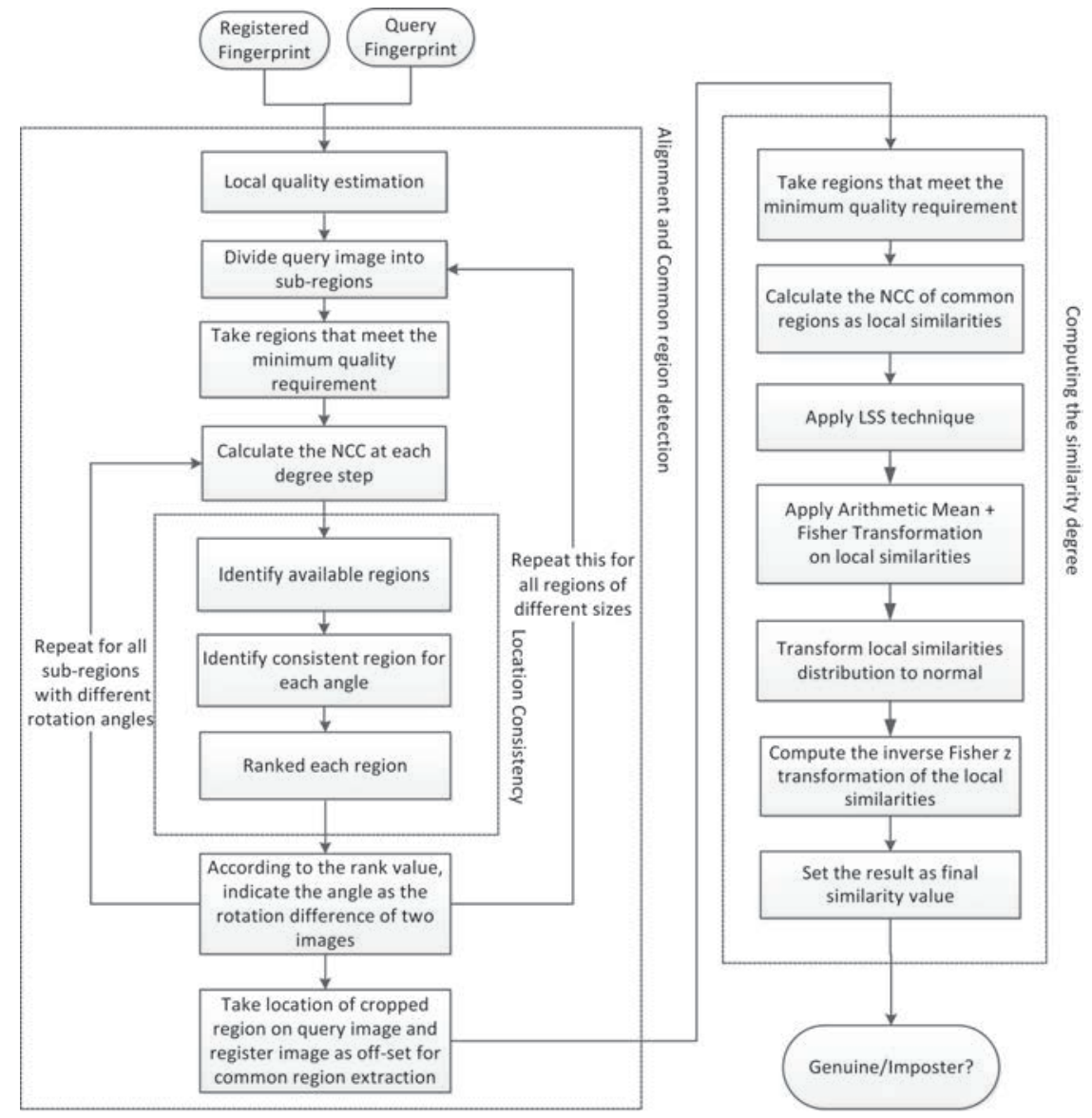

Figure 11 Final block diagram of the proposed partial fingerprint matching method.

FVC_2002_DB1, the participants who volunteered to provide their fingerprints, were asked to intentionally change the orientation of their finger on the scanner when scanning different impressions (to make the different impressions vary rotationally). No effort was made to control the image quality, the sensor plates were not systematically cleaned and high quality images were removed from the dataset. Due to rotation and displacement of the finger when scanning, there is often only a partial overlap between different impressions of the same finger and hence, the images in the data set include low quality and partial fingerprints as well. FVC_2002_DB1 dataset was obtained by using an older sensor compared to FVC_2004 and FVC_2006 
Table 4 Properties of datasets FVC_2002_DB1 and FVC_2006_DB2 used for evaluation

\begin{tabular}{lcc}
\hline Dataset & FVC_2002_DB1 & FVC_2006_DB2 \\
\hline Sensor Type & Optical Sensor & Optical Sensor \\
\hline Image Size & $388 \times 374$ & $400 \times 560$ \\
\hline Resolution & $500 \mathrm{dpi}$ & $569 \mathrm{dpi}$ \\
\hline Number of fingers & 100 & 140 \\
\hline Number of impressions per finger & 8 & 12 \\
\hline Total number of fingerprints & $100 \times 8$ & $140 \times 12$ \\
\hline
\end{tabular}

datasets. Due to the older technology, the quality of the images are low in this dataset. This data set is tediously used by researchers which makes comparing the results easier. Also it saves the effort of implementing others' works for comparison purposes.

Considering the number of impressions per finger, there are 2800 and 9240 intra comparisons for FVC_2002_DB1 and FVC_2006_DB2 datasets respectively. Since the number of inter comparisons is relatively large (compared to intra-comparison), only the first impression of each finger is compared with the first impression of other fingers. Thus, there are 4950 and 9730 inter comparisons for FVC_2002 DB1 and FVC_2006_DB2 datasets. The partial fingerprint datasets are generated from both datasets by only considering certain percentage of the fingerprint foreground area (Section 7). Therefore, the same number of inter and intra comparisons as original datasets are considered. The accuracy of the proposed method is computed as:

$$
\text { Accuracy }=\frac{C A+C R}{T G+T I} \times 100
$$

where $C A$ is the number of genuine comparisons that are correctly accepted, $C R$ is the number of imposter comparisons that are correctly rejected, $T G$ and $T I$ are the total number of genuine (intra) and imposter (inter) comparisons respectively. The error rate is computed as:

$$
\text { Error Rate }=\frac{F A+F R}{T G+T I} \times 100, \equiv 100-\text { Accuracy }
$$

where $F A$ and $F R$ refers to the number of falsely accepted and falsely rejected comparisons.

Table 5 shows the results of matching the fingerprints of dataset FVC_2002_DB1 and FVC_2006_DB2. The low error rate achieved is due to using all the available information and processing the local similarities to consolidate them into a final matching score as discussed. 
Table 5 Recognising all the fingerprints only based on Method 2

\begin{tabular}{lcc}
\hline & FVC_2002_DB1 & FVC_2006_DB2 \\
\hline FRR(\%) & 2.6429 & 2.5760 \\
\hline FAR(\%) & 2.6672 & 2.5535 \\
\hline EER(\%) & 2.6650 & 2.5648 \\
\hline Threshold $_{(E E R)}$ & 0.5450 & 0.4665 \\
\hline
\end{tabular}

Table 6 provides the comparison of the proposed method with previous works in terms of EER metric on the FVC_2002_DB1 dataset. Two different experiments has been done (i) only those fingerprints of database are considered which consist of at least one singulairty point (ii) whole dataset is considered. In first experiment all fingerprints have a singular point and since the area around the reference points provides more discriminative information than any other areas. Therefore, the proposed method could recognise fingerprints with $2.02 \%$ EER. However, for second experiment the proposed alignment method is used which only uses the fingerprint ridges and extracts common regions independent of reference point. Hence, proposed method results in EER of $2.665 \%$.

To demonstrate the effectiveness of the proposed region-based method, the result is compared with the single region-based correlation (conventional correlation-based approach). It was observed that the averaging method improved the EER from $7.1 \%$ to $2.665 \%$ with respect to the conventional

Table 6 Comparison of the proposed method with previous works in terms of EER(\%) value on the dataset FVC_2002_DB1. The methods are roughly categorised into the three major groups of fingerprint approaches

\begin{tabular}{lcc}
\hline Category & Method & EER (\%) \\
\hline Minutiae-Based & Kovacs-Vajna, 2000 [13] & 4.3 \\
& Tico, 2003 [14] & 4.0 \\
& Chen, 2005 [7] & 4.6 \\
& Liu, 2005 [15] & 4.3 \\
& Gao, 2011 [16] & 3.5 \\
\hline Non-Minutaie-Based & Sha, 2003 [17] & 6.23 \\
& Yang, 2007 [18] & 3.64 \\
& Lumini, 2006 [19] & 4.2 \\
& Qader, 2007 [20] & 7.13 \\
\hline Hybrid & Benhammadi, 2007 [21] & 4.2 \\
& Abraham, 2011 [22] & 0.75 \\
\hline Single Region-Based & Conventional & 7.1 \\
\hline Region-Based & Fingerprints with 1 singularity point & 2.02 \\
Methods & Full Dataset & 2.665 \\
\hline
\end{tabular}


single region-based method. This improvement is achieved due to the robustness of the proposed method in handling the non-linear distortion and lowering the effect of the distorted regions in the fingerprints.

In minutiae-based approaches, the method proposed by Gao et al. results in best EER that is 3.5. However, the method suffers from missing and spurious minutiae and also not working on all the available information of a fingerprint. The lowest EER in non-minutiae category belongs to Yang et al.'s [23] method. Yang et al.'s method is based on extracting invariant moments of the fingerprint. This method mainly suffers from two problems. The first problem is that they only make use of a small region of the fingerprints (around reference points) while the rest of the information remains unused. The second problem is that they used $75 \%$ of the dataset as training set, while all the dataset (including the training set) is used to evaluate their method (as test set). This significantly affects the performance of the system and leads to a lower EER compared to evaluating the system only on the test set.

On the other hand, in the proposed approach we conducted a pixel-wise comparison which is able to make use of level-3 features on the fingerprint which are not as sensitive as minutiae-based methods to the fingerprint quality. Hence, the proposed method uses all the available dimensional attributes of the fingerprint. Additionally, the proposed method is able to take into account all the possible distinguishing information in the fingerprint, regardless of the image quality (not dependent on few particular features). However, the lowest EER among all methods is achieved through using both minutiae and non-minutiae features of the fingerprint as proposed by Abraham et al. [22] by extracting the secondary features from minutiae information. They have used the minutiae points in addition to shape context and descriptors of the fingerprint. The EER obtained by their method is very low, though when the fingerprints are partial (Section 7) the accuracy is decreased.

Regarding the time cost of the proposed method, the matching process of two fingerprints takes about 1.5 minutes on a computer with $3.1 \mathrm{GHz} \mathrm{CPU}$ and 8 GB RAM. This time has a direct relationship with how partial a fingerprint is. The smaller the available valid regions are, the less time is required for the matching process. Considering the size of the dataset, the time cost of the proposed method, and the number of experiments conducted, the comparisons were done on a High Performance Computing Cluster. 


\section{Experimental Result on Partial Fingerprints}

In order to measure the effect of size of partial fingerprints, a series of partial fingerprints with different sizes from FVC_2002_DB1 and FVC_2006_DB1 are generated. The datasets are generated by considering different sizes (as percentage) of the fingerprint foreground area at random positions as in Jea and Govindaraju [3] and Vijayaprasad [24]. The region sizes considered are $20 \%, 30 \%, 40 \%, 50 \%, 60 \%, 70 \%$ and $80 \%$ of the foreground area of the fingerprint. These conditions are set to simulate where a partial fingerprint is provided.

For the dataset FVC_2002_DB1 and FVC_2006_DB2, when only a partial size of both registered and query fingerprints' foreground is considered, the result of the proposed method is compared with Abraham et al.'s method. The source code of their method is available at MathWork.com [25]. The performance of the proposed method is measured in terms of EER and number of cases processed by each method.

For the dataset FVC_2002_DB1, Table 7 shows that if less than $50 \%$ of the fingerprint foreground area is considered, the proposed method performs better than Abraham et al.'s method. The EER of the proposed method is lower than Abraham et al.'s method in almost all the cases when part of the fingerprint area is considered. Since there might be a small fingerprint area left after considering only a portion of the foreground area (the original fingerprint might be partial), some fingerprints provide limited information to be used by the matching method. Therefore, the number of cases that could be recognised is less than the total number of comparisons in the dataset. The number of cases that could be processed by the proposed method and Abraham et al.'s method are shown in Table 8 . The number of cases that are processed by the proposed method is higher or the same as in Abraham et al. method.

Table 7 Comparison of the performance of the partial fingerprint matching with different sizes at random position on dataset FVC_2002_DB1 in terms of EER

\begin{tabular}{lcc}
\hline$\%)$ & \multicolumn{2}{c}{ EER (\%) } \\
\hline Image Size & Abraham [22] & Proposed Method \\
\hline 80 & 3.2643 & 2.8546 \\
\hline 70 & 3.5076 & 3.2150 \\
\hline 60 & 3.4203 & 3.7653 \\
\hline 50 & 5.4147 & 4.5014 \\
\hline 40 & 5.8945 & 5.7217 \\
\hline 30 & 8.3258 & 7.2311 \\
\hline 20 & 14.6893 & 12.4027 \\
\hline
\end{tabular}


Table 8 Sensitivity analysis of the partial fingerprint matching methods w.r.t. the image size in terms of the percentage of the cases that each method was able to proceed when only partial size of the fingerprint foreground area was considered

\begin{tabular}{lcc}
\hline$(\%)$ & Percentage of the Cases Processed. Total \\
\hline Intra, Inter $)$ \\
\hline 80 & Abraham [22] & Proposed Method \\
\hline 70 & $7749_{(2799,4950)} \simeq 100 \%$ & $7746_{(2797,4949)} \simeq 100 \%$ \\
\hline 60 & $7750_{(2800,4950)} \simeq 100 \%$ & $7746_{(2798,4948)} \simeq 100 \%$ \\
\hline 50 & $5254_{(1432,3822)} \simeq 68 \%$ & $7732_{(2784,4948)} \simeq 100 \%$ \\
\hline 40 & $4145_{(1068,3077)} \simeq 53 \%$ & $7692_{(2745,4947)} \simeq 99 \%$ \\
\hline 30 & $3181_{(784,2397)} \simeq 41 \%$ & $7666_{(2717,4949)} \simeq 99 \%$ \\
\hline 20 & $1105_{(245,860)} \simeq 14 \%$ & $7382_{(2478,4904)} \simeq 95 \%$ \\
\hline
\end{tabular}

As represented in Table 9, the EER of Abraham et al.'s method is very high for FVC_2006_DB2 dataset when fingerprints are of partial size. The reasons for the high error rate of their method is that the parameters are tuned for the FVC_2002_DB1 dataset without changing the parameters for a different dataset their method produces a very high error rate. In addition, as the sensitivity analysis shows (Table 10), the number of cases processed in both methods is higher than the dataset FVC_2002_DB1.

Table 9 Comparison of the performance of the partial fingerprint matching with different sizes at random positions on dataset FVC_2006_DB1 in terms of EER

\begin{tabular}{lcc}
\hline$(\%)$ & \multicolumn{2}{c}{ EER (\%) } \\
\hline Image Size & Abraham [22] & Proposed Method \\
\hline 100 & 11.0921 & 2.56 \\
\hline 80 & 41.0636 & 3.3150 \\
\hline 60 & 37.3257 & 3.6271 \\
\hline 40 & 31.5654 & 4.3497 \\
\hline 20 & 21.63 & 5.1945 \\
\hline
\end{tabular}

Table 10 Sensitivity analysis of the partial fingerprint matching methods w.r.t. of the image size in terms of the percentage of the cases that each method was able to processed when only a partial size of the fingerprint foreground area was considered. (FVC_2006_DB2)

\begin{tabular}{lcc}
\hline$(\%)$ & \multicolumn{2}{c}{ Percentage of the Cases Processed. Total } \\
$($ Intra,Inter $)$ \\
\hline Image Size & Abraham [22] & Proposed Method \\
\hline 80 & $18970_{(9240,9730)} \simeq 100 \%$ & $18884_{(9224,9660)} \simeq 100 \%$ \\
\hline 60 & $18970_{(9240,9730)} \simeq 100 \%$ & $18885_{(9220,9665)} \simeq 100 \%$ \\
\hline 40 & $18634_{(9078,9556)} \simeq 98 \%$ & $18578_{(9155,9423)} \simeq 98 \%$ \\
\hline 20 & $12023_{(5817,6216)} \simeq 63 \%$ & $11103_{(6155,4948)} \simeq 58 \%$ \\
\hline
\end{tabular}


This is due to the higher resolution and image size in FVC_2006_DB1 dataset. Also, both methods can cover almost $98 \%$ of the comparisons in this dataset due to the large fingerprint size and high resolution when $40 \%$ or more of the foreground area is considered.

To recapitulate, the proposed fingerprint recognition method performs better than other related works in the literature, particularly, when fingerprints are partial.

\section{Conclusion}

Alignment is one the most important phases in fingerprint matching. However, aligning the fingerprints specially the partial fingerprints is very difficult by using the existing alignment techniques. A region-based alignment method is proposed that can align fingerprints with or without using the singularities in fingerprints. Moreover, for region-based matching, the accurate alignment is very crucial, therefore ranking and quality of the regions is also used. The information obtained through alignment, is used not only to recognise fingerprints but also to identify their common regions. Recognising fingerprints with a high degree of confidence at this level not only reduces the complexity of matching but also results in high recognition accuracy.

For complete matching process, the common regions are extracted by using the location of regions resulting in highest ranking as the offset point. In order to measure the final similarity in such a way that it better distinguishes the intra and inter cases (considering high intra variation and inter similarity in some cases), different techniques are investigated. Global consolidation techniques are applied to ensure selecting the most appropriate local similarities according to the quality, adjacent regions and global structure of the fingerprints. Also, the arithmetic mean of the local similarity is used for computing a global similarity value between the fingerprints.

The experiments are conducted on FVC datasets and compared with other existing methods. The results show that the proposed method performs better in most of the cases when compared with other methods. To sum up, the proposed method which directly uses the fingerprint texture information, provides a simple but effective method to recognise fingerprints. Using other fingerprint features with the proposed approach can improve the accuracy of recognition even more. This approach is applicable to partial and low quality fingerprints as well where most of the existing fingerprint matching techniques do not perform well. 


\section{References}

[1] Zhao, Q., Zhang, D., Zhang, L., and Luo, N. (2010). High resolution partial fingerprint alignment using pore-valley descriptors. Pattern Recognition, 43(3), 1050-1061.

[2] Khalil, M. S., Muhammad, D., Khan, M. K., and Qais, A. N. (2009). Fingerprint verification using fingerprint texture. In International Symposium on Signal Processing and Information Technology (ISSPIT), 591-594. doi:10.1109/ISSPIT.2009.5407549

[3] Jea, T. Y., and Govindaraju, V. (2005). A minutia-based partial fingerprint recognition system. Pattern Recognition, 38(10), 1672-1684.

[4] Maltoni, D., Maio, D., Jain, A. K., and Prabhakar, S. (2009). Handbook of Fingerprint Recognition, 2nd Edition, Springer: New York.

[5] Wang, L., Bhattacharjee, N., and Srinivasan, B. (2011). A novel technique for singular point detection based on poincaré index. In Proceedings of the 9th International Conference on Advances in Mobile Computing and Multimedia, 12-18.

[6] Bennamoun, M. and Mamic, G. J. (2002). Object Recognition: Fundamentals and Case Studies, Springer.

[7] Chen, Y., Dass, S. C., Jain, A. K. (2005). Fingerprint Quality Indices for Predicting Authentication Performance, Lecture Notes in Computer Science, Springer, 160-170.

[8] Cappelli, R., Ferrara, M., and Maltoni, D. (2010). Minutia cylinder-code: A new representation and matching technique for fingerprint recognition. IEEE Transactions on Pattern Analysis and Machine Intelligence, 32(12), 2128-2141.

[9] Yoo, J. C., and Han, T. H. (2009). Fast normalized cross-correlation. Circuits, Systems and Signal Processing, 28(6), 819-843.

[10] Agarwal, B. (2007). Programmed Statistics (Question-Answers), 2nd Edition, New Age International Ltd.

[11] Fisher, R. A. (1921). On the probable error of a coefficient of correlation deduced from a small sample. Metron, 1, 3-32.

[12] Maio, D., Maltoni, D., Cappelli, R., Wayman, J. L., and Jain, A. K. (2002). FVC_2002: Second fingerprint verification competition. In Proceeding of the 16th International Conference on Pattern Recognition, 3, 811-814.

[13] Kovacs-Vajna, Z. M. (2000). A fingerprint verification system based on triangular matching and dynamic time warping. IEEE Transactions on Pattern Analysis and Machine Intelligence, 22(11), 1266-1276. 
[14] Tico, M., and Kuosmanen, P. (2003). Fingerprint matching using an orientation-based minutia descriptor. IEEE Transactions on Pattern Analysis and Machine Intelligence, 25(8), 1009-1014.

[15] Liu, N., Yin, Y., and Zhang, H. (2005). A fingerprint matching algorithm based on Delaunay triangulation net. In the Fifth International Conference on Computer and Information Technology (CIT), 591-595.

[16] Gao, Z., You, X., Zhou, L., and Zeng, W. (2011). A novel matching technique for fingerprint recognition by graphical structures. In International Conference on Wavelet Analysis and Pattern Recognition (ICWAPR), 77-82.

[17] Sha, L., Zhao, F., and Tang, X. (2003). Improved fingercode for filterbank-based fingerprint matching. In International Conference on Image Processing (ICIP), 2, 895-898.

[18] Zhang, Y., Yang, X., Su, Q., and Tian, J. (2007). Fingerprint Recognition Based on Combined Features, Vol. 4642 of Lecture Notes in Computer Science, Springer: Berlin, Heidelberg, 281-289.

[19] Lumini, A., and Nanni, L. (2006). Two-class fingerprint matcher. Pattern Recognition, 39(4), 714-716.

[20] Qader, H. A., Ramli, A. R., and Al-Haddad, S. (2007). Fingerprint Recognition Using Zernike Moments. Int. Arab J. Inf. Technol., 4(4), 372-376.

[21] Benhammadi, F., Amirouche, M. N., Hentous, H., Beghdad, K. B., and Aissani, M. (2007). Fingerprint matching from minutiae texture maps. Pattern Recognition, 40(1), 189-197.

[22] Abraham, J. Gao, J. Kwan, P. (2011). Fingerprint Matching Using a Hybrid Shape and Orientation Descriptor, INTECH Open Access Publisher.

[23] Yang, J. C., Shin, J. W., and Park, D. S. (2006). Fingerprint matching using invariant moment features. In International Conference on Computational and Information Science, (pp. 1029-1038). Springer: Berlin, Heidelberg.

[24] Vijayaprasad, P., Sulaiman, M. N., Mustapha, N., and Rahmat, R. W. O. K. (2010). Partial fingerprint recognition using support vector machine. Information Technology Journal, 9(4), 844-848.

[25] Abraham, J. Fingerprint matching algorithm using shape context and orientation descriptors, access date: 14 March 2015 (2011). Available at: http://au.mathworks.com 


\section{Biographies}

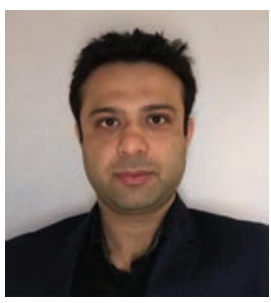

Omid Zanganeh received his Ph.D. degree in Computer Science from Monash University, Australia in 2016; M.Sc. degree in Computer Science from University of Technology Malaysia (UTM), Malaysia in 2011; and B.Sc. degree in Software Engineering from Azad University, Iran in 2008.

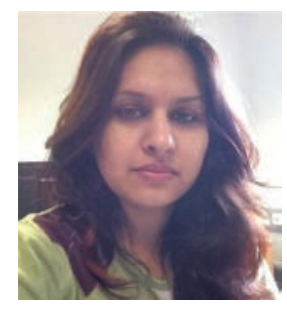

Komal Komal is a Ph.D. student in Faculty of Information Technology, Monash University, Australia. She has Masters of technology from the National Institute of technology, Kurukshetra, India and Bachelor of Engineering degree from Deenbandhu Chhotu Ram University of Science and Technology, India. Both of her Bachelors and Masters degree are in Electronics and Communications Engineering. Her main research interests are machine learning and computer vision. 


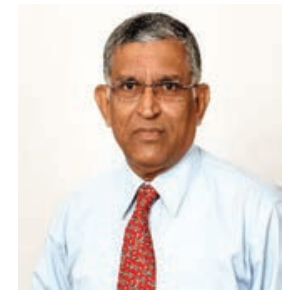

Bala Srinivasan is a professor of information technology in the Faculty of Information Technology, Monash University, Australia. He has more than 30 years of experience in academia, industries and research organizations. He has authored and jointly edited technical books and published in international journals and conferences in the areas of multimedia databases, data communications, data mining and distributed systems. He is a gold medallist in Bachelor of Engineering Honours degree in Electronics and communication Engineering, Guindy Engineering College, University of Madras, India, a Master's and a Ph.D. degree both in Computer Science from the Indian Institute of Technology, Kanpur, India.

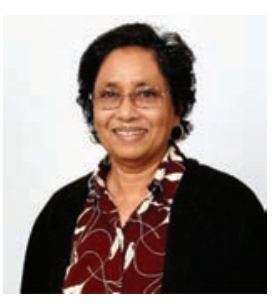

Nandita Bhattacharjee works in the Faculty of Information Technology at Monash University as a Senior Lecturer. Her main research areas are Advanced Implementation Systems, Audio Watermarking, Computer Systems Security, Computer vision, image and signal processing, Digital Design, Hardware Implementation of Neural Networks. 


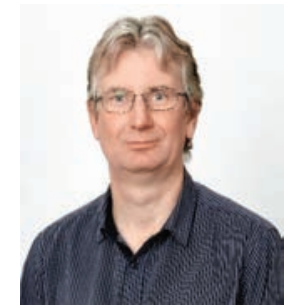

David Albrecht started in the Faculty of Information Technology in 1994. He has done research in several areas including Machine Learning, Bayesian Networks, User Modelling, Geostatistics, Logic, Functional Operator Theory, and Statistics. He is currently jointly supervising three $\mathrm{PhD}$. students and two Masters students. He has two honours degrees and a $\mathrm{PhD}$ in Mathematics from Monash (1994). During his time at Monash David has run an extension program for Primary School students, the Faculty Extension program for Secondary School students, worked with the teachers at John Monash Science School in developing several subjects, developed a unit which has become the basis of a new VCE unit, and was involved in the Thailand-Australia Science and Engineering Assistance Project (TASEAP) to improve the quality of undergraduate science and engineering in Thai universities. In 2015 he was nominated by the Faculty to be the Faculty Foundation Fellow of the Monash Education Academy. David is an experienced Coordinator and manager of courses, and is currently the chair of Faculty Academic Progress Committee. 
